\title{
Festbeträge - ein schlechtes Rezept für die Patientensicherheit
}

\section{Gert Printzen}

Dr. med., Mitglied des Zentralvorstandes der FMH, Departementsverantwortlicher Heilmittel

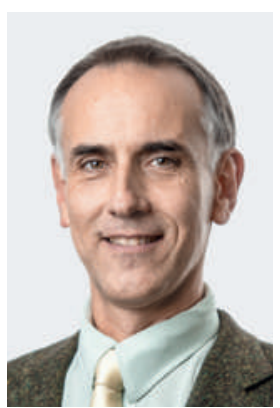

Auf Ende dieses Jahres hat der Bundesrat eine Änderung im Preissystem für patentfreie Medikamente angekündigt, die auf die ärztliche Tätigkeit nicht ohne Folgen bliebe: Neu sollen Referenzpreise pro Wirkstoff eingeführt werden. Die Versicherer würden dann nur noch diesen jeweils definierten Festbetrag bezahlen, egal für welches Medikament sich Arzt, Apotheker oder Patient entscheiden. Ist das bevorzugte Präparat teurer, muss der Patient die Differenz zum Festbetrag selbst bezahlen.

Der Preisüberwacher erwartet durch diesen Ansatz Einsparungen von mehreren 100 Mio. Franken und fordert ein Billigstpreissystem, bei dem der Festbetrag ganz simpel beim günstigsten Generikum angesetzt würde. Der Patient erhielte nur noch das Billigste ohne Aufzahlung oder anders ausgedrückt: Die Wahlfreiheit würde de facto entfallen.

\section{Wer ein Medikament auf seinen Wirkstoff} reduziert, setzt die Therapietreue aufs Spiel und nimmt die Verwechslungsgefahr in Kauf.

Das Festbetragssystem geht unseres Erachtens vom falschen Konzept aus, dass Medikamente mit gleichen Wirkstoffen identisch sind. Eine wesentliche Rolle spielen aber auch patientengerechte Darreichungsformen, verwechslungssichere Verpackungen und ergänzende Massnahmen zur Verbesserung der Compliance. Zudem ist der Therapieprozess nicht abgeschlossen, wenn der Arzt den Wirkstoff ausgewählt hat. Erst wenn der Patient sein Medikament versteht, darauf vertraut und es korrekt und zuverlässig einnimmt, kann der Arzneistoff wirken.

Will oder kann der Patient die Differenz vom Festbetrag zum optimalen oder Wunschpräparat nicht bezahlen, dann bekommt er nicht mehr das Medikament, das er kennt und dem er vertraut. Erzwungene Wechsel führen nachweislich zu einer geringeren Therapietreue und zu Verwechslungen mit teils erheblichen Folgekosten.

Heute entscheiden Ärzte und Apotheker auf der Basis fachlicher Überlegungen, welches Präparat für welchen Patienten geeignet ist. Ihre Wahlfreiheit ist im Rah- men der Spezialitätenliste nicht ökonomisch eingeschränkt, und sie können individuell das jeweils optimale Produkt verordnen bzw. abgeben. Das wäre bei einem Festbetragsmodell nicht mehr der Fall, weil dabei eindeutig der Preis zum bestimmenden Kriterium würde.

Ein Festbetragssystem verhindert zudem die Innovation im patentfreien Bereich. Wer wäre noch an der Entwicklung verbesserter, patientenfreundlicherer Darreichungsformen interessiert? Unter solchen Voraussetzungen lohnen sich die Entwicklungskosten nicht, weil keine Rentabilität mehr gegeben ist. Des Weiteren kann sich kein Anbieter mehr über Informationen und Service gegenüber seinen Konkurrenten abheben, weil auch dieser Mehrwert bei einem Festbetragssystem nicht abgegolten wird.

Laut einer kürzlichen Studie von Dichter Research steht die Ärzteschaft dem Festbetragssystem skeptisch gegenüber. Die Kolleginnen und Kollegen führen vorwiegend negative Auswirkungen an, z.B. ein hoher Mehraufwand für Erklärungen an die Patienten oder auch das Risiko von Mehrkosten aufgrund aufgezwungener Medikamentenwechsel, die vermehrt Konsultationen und Hospitalisationen wegen mangelnder Compliance zur Folge haben.

\section{Versorgungsqualität und Innovation sind gefährdet.}

Die heute geltenden Regelungen im patentfreien Bereich (dynamisierter differenzierter Selbstbehalt) wurden erst 2012 eingeführt und verdienen nach unserer Meinung eine Chance, sich zu bewähren. Erste Daten zeigen, dass sie zu einem verstärkten Preiswettbewerb mit daraus resultierenden Kosteneinsparungen für die soziale Krankenversicherung führen. Der Patient wird auch hier in die ökonomische Pflicht genommen, allerdings in einer sozial zumutbaren und abgefederten Form über eine moderate Erhöhung des Selbstbehaltes. Festbeträge nach dem Billigstsystem sind keine sinnvolle Alternative zum bestehenden System, geschweige denn eine sozialverträgliche Sparmassnahme. 